\title{
Achieving Cooperative Diversity with Multiple Frequency Offset Estimation
}

\author{
Ali A. Nasir, Salman Durrani and Rodney A. Kennedy \\ School of Engineering, CECS, The Australian National University, Canberra, Australia. \\ Email: \{ali.nasir, salman.durrani, rodney.kennedy\}@anu.edu.au
}

\begin{abstract}
In cooperative communications, estimation and compensation of multiple carrier frequency offsets is an important implementation issue that needs to be addressed in practice. This paper proposes an efficient maximum likelihood estimator (MLE) using alternating projection technique, for the joint estimation of Rayleigh fading channels and multiple carrier frequency offsets (MCFO) in cooperative communication systems. Our proposed equalizer incorporates these channel and MCFO estimates and uses matrix inverse technique to decode the transmitted symbols. Unlike existing schemes for equalizer design, the proposed design does not assume any perfect MCFO and/or channel estimate knowledge at the receiver or code redundancy or delay diversity and works for the full range $( \pm 0.5$ cycles/sec) of the normalized frequency offsets. We present simulation results to illustrate that the Mean Square Error (MSE) of MCFO achieves the Cramér Rao Bound (CRB) above $7 \mathrm{~dB}$ signal-to-noise ratio (SNR) while the MSE of channel estimation achieves the CRB for all SNR values. In addition, the Bit Error Rate (BER) performance shows that our proposed equalizer can achieve the full spatial diversity gain of space-time block codes.
\end{abstract}

\section{INTRODUCTION}

Cooperative diversity for wireless networks has been proposed to enable distributed nodes with single antennas in a wireless network to form virtual antenna arrays and to achieve the improved performance benefits as in conventional multiple antenna systems [1]. In cooperative communications, multiple transmissions from relays at different locations with individual local oscillators gives rise to multiple timing and multiple carrier frequency offsets (MCFO) that need to be compensated for at the receiver node. Assuming perfect timing and frequency synchronization at the relays, conventional space-time coding techniques can be applied to decode and forward cooperative communication systems to achieve the benefits of cooperative diversity [1]. Thus time and frequency synchronization is an important implementation issue needed to be addressed in practice. When there are multiple timing offsets from relay nodes, there have been several studies in the literature showing how to achieve the asynchronous cooperative diversity [2], [3]. In this paper, our focus is on achieving cooperative diversity in the presence of multiple carrier frequency offsets.

Assuming MCFO are somehow perfectly estimated, equalizer design for MCFO compensation has been targeted for various cooperative communication scenarios [3]-[7]. Distributed linear convolutive space-time codes are exploited for equalizer design in [3]. However the design in [3] uses delay diversity and zero padding which affects the data rate. Space-
Time Block Code (STBC) and Space-Frequency Block Code (SFBC) with Orthogonal Frequency Division Multiplexing (OFDM) is employed in [4] and [5] respectively. The authors in [4] assume prior coarse frequency synchronization and their equalization works only under a small range of carrier offsets i.e., $\pm 0.05 \times$ subcarrier spacing. Similarly the MCFO compensation proposed by [5] works only if carrier offset is within $\pm 0.1 \times$ subcarrier spacing. Minimum Mean Square Error Decision Feedback Equalizer (MMSE-DFE) is proposed by [6], but reliable performance requires delay diversity and normalized frequency offset to be less than $10^{-6}$ cycles per sample, which is a very small acquisition range. SFBC with OFDM is also employed in the equalizer design of [7], but the solution relies on the specific structure of OFDM systems. All the schemes in [3]-[7], focus on MCFO compensation and do not address MCFO estimation.

Recent papers in the literature have considered this difficult problem of estimation of MCFO using both pilot symbols [8][11] and blind approaches [12], [13]. In [8], a maximum likelihood estimation algorithm is proposed but its computational complexity is very high due to a multi-dimensional minimization requirement. The authors in [8] propose to reduce the computational complexity by advising the strategy where one transmitter antenna transmits a training symbol while the others remain silent, but this design lowers the data rate. In [9], the authors propose a correlation based estimator (CBE) by transmitting orthogonal training sequences from different antennas but the scheme suffers from an error floor. An iterative scheme for eliminating the error floor of [9] is proposed in [10], but the estimator performs poorly for normalized frequency offset greater than \pm 0.05 , as the orthogonality of training sequences is not preserved. This assumption of small MCFO is generally not justifiable for cooperative systems due to the distributed location of nodes and their independent oscillators [11]. Iterative MCFO estimation based on MUltiple SIgnal Characterization (MUSIC) algorithm is proposed in [11] and shown to outperform existing algorithms at mid-to-high signal to noise ratios. However the accuracy of the estimation depends on the threshold value set between the likelihood function of two iterations. A blind method of MCFO estimation is proposed in [12] based on formulating a virtual MIMO system using polyphase components of the oversampled signal. In [13], the approach is shown to be able to separate two users in a distributed Multiple Input Single 
Output (MISO) system. However, for more than two users the oversampling requirements become prohibitive and the approach becomes impractical.

In this paper, we assume that there are no timing errors and consider the problem of both MCFO estimation and compensation. We propose a maximum likelihood estimator using alternating projection for joint estimation of Rayleigh fading channels and Multiple Carrier Frequency Offsets (MCFO) in cooperative communication systems. We use the alternating projection technique in [14] to reduce the computational complexity and convert the multi-dimensional minimization task into a series of simpler 1-dimensional minimization problems. Our proposed equalizer design incorporates the channel and MCFO estimates and uses matrix inverse technique to estimate the symbols. Our equalizer works for the full acquisition range $( \pm 0.5$ cycles/sec) of the normalized frequency offsets without the need of any code redundancy or delay diversity. We state the closed form Cramér Rao Bound (CRB) for the estimation problem and show that it can be obtained as a special case of the result in [8]. We show that the Mean Square Error (MSE) of MCFO and channel estimates achieves the Cramér Rao Bound (CRB) and the Bit Error Rate (BER) performance achieves the full spatial diversity gain offered by space-time block codes.

This paper is organised as follows. The physical layer signal model is provided in Section II. The procedure for joint estimation of MCFO and channels is described in Section III. The proposed equalizer design is explained in Section IV. The CRB analysis is discussed in Section V. These bounds are compared to the simulation results in Section VI. Finally, conclusions are drawn in Section VII.

\section{System ModeL}

We consider a decode and forward cooperative communication system with one source node, one destination node and $R$ relay nodes. All the nodes are equipped with a single antenna. We assume perfect error-free transmission from source to relay nodes. ${ }^{1}$ For simplicity, we consider $R=2$ relays cooperating to transmit the source information. Thus the simplified system model considered in this paper is as shown in the Fig. 1. We assume perfect symbol synchronism among the relays and destination. The flat-fading channel coefficient from Relay $R_{k}$ to destination node is $h_{k}$, where $k=\{1,2\}$ is the relay index. These coefficients are modeled as zero-mean complex Gaussian random variables with unit variance, leading to a Rayleigh fading channel model. In addition, we assume quasistatic fading coefficients, i.e., all the fading coefficients are constant for a frame of transmitted bits but are independent and identically (i.i.d.) distributed from frame to frame. The transmission frame length is $2 N$ and consists of two $N$ symbol periods: (i) training session and (ii) data transmission phase. The system model description for the two intervals is described in the following subsections.

\footnotetext{
${ }^{1}$ This assumption is used in this work since standard techniques are available in the literature for the estimation of a single timing and a single carrier offset in each source to relay link [15].
}

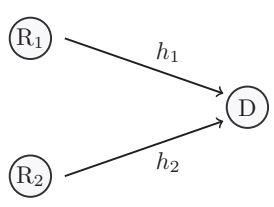

Fig. 1. Block diagram for cooperative communication system.

\section{A. Training Period}

In the first phase, the two relays transmit distinct and uncorrelated symbols as training sequences of length $N$ over flat Rayleigh fading channel. The $n^{\text {th }}$ received symbol at the destination node can be written as

$$
y_{t}(n)=\sum_{k=1}^{2} h_{k} e^{j 2 \pi f_{k} n} x_{k}(n)+w(n),
$$

where $y_{t}$ corresponds to the received sequence during training session, $h_{k}$ is the Rayleigh flat-fading channel from relay $R_{k}$ to the destination node, $w(n)$ is the Additive White Gaussian Noise (AWGN) with variance $\sigma_{w}^{2}, x_{k}(n)$ is the $n^{\text {th }}$ training symbol from the $k^{\text {th }}$ relay, $n=\{1,2, \ldots, N\}, N$ is the length of training sequence and $f_{k}$ is the digital frequency offset between relay $R_{k}$ and the destination node, in cycles/sample.

At the destination, we perform joint estimation of multiple frequency offsets and channels with the help of known pilot sequences. The vector representation of (1) can be given as

$$
\mathbf{y}_{t}=\left[\begin{array}{ll}
\mathbf{E}_{1} \mathbf{x}_{1} & \mathbf{E}_{2} \mathbf{x}_{2}
\end{array}\right] \mathbf{h}+\mathbf{w}=\boldsymbol{\Omega}_{\mathbf{f}} \mathbf{h}+\mathbf{w},
$$

where

$$
\begin{aligned}
\mathbf{y}_{t} & \triangleq\left[y_{t}(1), y_{t}(2), \ldots, y_{t}(N)\right]^{T} \\
\mathbf{\Omega}_{\mathbf{f}} & \triangleq\left[\mathbf{E}_{1} \mathbf{x}_{1} \quad \mathbf{E}_{2} \mathbf{x}_{2}\right] \\
\mathbf{E}_{k} & \triangleq \operatorname{diag}\left(\left[e^{j 2 \pi f_{k}(1)}, e^{j 2 \pi f_{k}(2)}, \ldots, e^{j 2 \pi f_{k}(N)}\right]\right) \\
\mathbf{x} & \triangleq[x(1), x(2), \ldots, x(N)]^{T} \\
\mathbf{w} & \triangleq[w(1), w(2), \ldots, w(N)]^{T}
\end{aligned}
$$

where the superscript $(\cdot)^{T}$ denotes the transpose operator, $\mathbf{h}=[h(1), h(2)]^{T}, \mathbf{f}=[f(1), f(2)]^{T}$ and $\operatorname{diag}(\cdot)$ represents a diagonal matrix.

\section{B. Data transmission Period}

After the training period, the relays transmit the data sequence cooperatively by exploiting the $2 \times 1$ Alamouti Space-Time Block Code (STBC) [16]. Let us assume $\mathbf{s}=$ $[s(1), s(2), \ldots, s(N)]^{T}$ is the desired data sequence to be transmitted. If the relay $R_{1}$ transmits $s(n)$ and relay $R_{2}$ transmits $s(n+1)$ at the $n^{\text {th }}$ instant, then relay $R_{1}$ will transmit $-s^{*}(n+1)$ and relay $R_{2}$ will transmit $s^{*}(n)$ at the $(n+1)^{\text {th }}$ time instant. We can modify (2) to write the system model equation for data transmission phase as

$$
\mathbf{y}_{d}=\left[\begin{array}{ll}
\mathbf{E}_{1} \mathbf{s} & \mathbf{E}_{2} \mathbf{s}^{\prime}
\end{array}\right] \mathbf{h}+\mathbf{w},
$$

where $\mathbf{y}_{d}$ denotes the received sequence during data transmission phase. The modified vector 
$\mathbf{s}^{\prime}=\left[s^{\prime}(1), s^{\prime}(2), \ldots, s^{\prime}(N)\right]^{T}$ depends on the data sequence $\mathbf{s}$ as

$$
s^{\prime}(n)=\left\{\begin{array}{rrr}
-s^{*}(n+1), & \forall \text { odd } n \\
s^{*}(n-1), & \forall \text { even } n
\end{array}\right.
$$

where superscript $(\cdot)^{*}$ denotes the conjugate operator. The estimation of the channel and multiple frequency offsets and equalizer design is described in the following sections.

\section{Estimation of Multiple Frequency OfFsets And CHANNEL}

The joint likelihood function of the frequency offsets and channels can be derived using (3) as

$$
p\left(\mathbf{y}_{t} ; \mathbf{f}, \mathbf{h}\right)=\left(\pi \sigma_{w}^{2}\right)^{-N} \exp \left\{-\frac{\left\|\mathbf{y}_{t}-\mathbf{\Omega}_{\mathbf{f}} \mathbf{h}\right\|^{2}}{\sigma_{w}^{2}}\right\}
$$

Maximizing the above function is equivalent to minimizing the following cost function [8]

$$
J(\mathbf{f}, \mathbf{h})=\left\|\mathbf{y}_{t}-\boldsymbol{\Omega}_{\mathbf{f}} \mathbf{h}\right\|^{2}
$$

It can be readily shown that for a constant $f$, the maximum likelihood estimate of the unknown channel vector $\mathbf{h}$ is [8]

$$
\hat{\mathbf{h}}=\left(\boldsymbol{\Omega}_{\mathbf{f}}^{H} \boldsymbol{\Omega}_{\mathbf{f}}\right)^{-1} \boldsymbol{\Omega}_{\mathbf{f}}^{H} \mathbf{y}_{t}
$$

where the superscript $(\cdot)^{H}$ denotes the hermitian operator. After substituting (7) in (6), a cost function that only depends on $\mathbf{f}$ is obtained as

$$
\Lambda(\mathbf{f})=\left\|P_{\boldsymbol{\Omega}}^{\perp}(\mathbf{f}) \mathbf{y}_{t}\right\|^{2}
$$

where $P_{\boldsymbol{\Omega}}^{\perp}(\mathbf{f})=\mathbf{I}-P_{\boldsymbol{\Omega}}(\mathbf{f}), P_{\boldsymbol{\Omega}}(\mathbf{f})=\boldsymbol{\Omega}_{\mathbf{f}}\left(\boldsymbol{\Omega}_{\mathbf{f}}^{H} \boldsymbol{\Omega}_{\mathbf{f}}\right)^{-1} \boldsymbol{\Omega}_{\mathbf{f}}^{H}$. Then the multiple frequency offsets $\mathbf{f}$ are estimated as

$$
\hat{\mathbf{f}}=\arg \min _{\mathbf{f}} \Lambda(\mathbf{f})
$$

In the above minimization problem in (9), there are multiple frequency offsets from two relay-destination pairs, which requires an exhaustive search over a 2-dimensional space. In general for $M$ relays, this becomes a multi-dimensional minimization task whose computational complexity is huge. To reduce the computational complexity at the destination node, we use alternating projection [14], which reduces the $M$-dimensional minimization into a series of 1-dimensional minimization problems. The procedure consists of cycles and steps. In our case, a cycle is made of $M=2$ steps, and each step updates the CFO of a single user while keeping the other $\mathrm{CFO}$ constant at its most updated value. Note that we achieve optimal estimation in just 3 cycles. Further details on the implementation of alternating projection are elaborated in [14]. Finally, the channels are estimated using (7).

\section{Proposed Equalizer Design}

The proposed equalizer at the destination decodes the transmitted data sequence with the help of frequency and channel estimates obtained in the previous training period. The noise free received data symbol at $n^{\text {th }}$ and $(n+1)^{\text {th }}$ instant can be written as

$$
\begin{aligned}
y_{d}(n) & =h_{1} e^{j 2 \pi f_{1} n} s(n)+h_{2} e^{j 2 \pi f_{2} n} s(n+1) \\
y_{d}(n+1) & =-h_{1} e^{j 2 \pi f_{1}(n+1)} s^{*}(n+1)+h_{2} e^{j 2 \pi f_{2}(n+1)} s^{*}(n)
\end{aligned}
$$

Taking the conjugate of (11) will result in

$y_{d}^{*}(n+1)=-h_{1}^{*} e^{-j 2 \pi f_{1}(n+1)} s(n+1)+h_{2}^{*} e^{-j 2 \pi f_{2}(n+1)} s(n)$

Writing (10) and (12) in the matrix form, we obtain,

$$
\left[\begin{array}{c}
y_{d}(n) \\
y_{d}^{*}(n+1)
\end{array}\right]=\left[\begin{array}{cc}
A & B \\
C & D
\end{array}\right]\left[\begin{array}{c}
s(n) \\
s(n+1)
\end{array}\right]
$$

where $A=h_{1} e^{j 2 \pi f_{1} n}, B=h_{2} e^{j 2 \pi f_{2} n}, C=h_{2}^{*} e^{-j 2 \pi f_{2}(n+1)}$ and $D=-h_{1}^{*} e^{-j 2 \pi f_{1}(n+1)}$. Using the multiple frequency offsets and channel estimates as described in Sec. III, the equalized data symbols can be calculated by taking inverse of (13) as

$$
\left[\begin{array}{c}
\hat{s}(n) \\
\hat{s}(n+1)
\end{array}\right]=\frac{1}{R}\left[\begin{array}{cc}
D & -B \\
-C & A
\end{array}\right]\left[\begin{array}{c}
y_{d}(n) \\
y_{d}^{*}(n+1)
\end{array}\right]
$$

where $R=-\left|h_{1}\right|^{2} e^{-j 2 \pi f_{1}}-\left|h_{2}\right|^{2} e^{-j 2 \pi f_{2}}$. Thus transmitted data symbols are decoded pairwise using (14).

It must be noted that the proposed design requires taking equalization matrix inverses for every two symbols. This is a common requirement in the equalization literature [4]-[6], where the equalization matrix inverse often needs to be retaken every symbol (or OFDM symbol) even in a channel coherent duration. Using a similar analogy and higher-order STBC, the above equalizer design can be easily extended to cope with larger number of relays and their frequency offsets.

\section{Cramér RaO Bound Analysis}

In this section, we formulate the joint Cramér Rao Bound (CRB) for estimation of multiple frequency offsets and channel estimates. The received vector in (3) at the destination has circular symmetric complex normal distribution given as

$$
\mathbf{y}_{\mathbf{t}} \propto \mathcal{C N}\left(\boldsymbol{\mu}_{\mathbf{y}}, \sigma_{w}^{2} \mathbf{I}\right)
$$

where $\boldsymbol{\mu}_{\mathbf{y}}=\boldsymbol{\Omega}_{\mathbf{f}} \mathbf{h}$ and $\mathbf{I}$ is an $N \times N$ identity matrix.

Let $\boldsymbol{\theta} \triangleq\left[\Re(\mathbf{h})^{T}, \Im(\mathbf{h})^{T}, \mathbf{f}^{T}\right]^{T}$ be the vector of parameters of interest. The Fisher Information Matrix (FIM) $\mathbf{F}$ of $\boldsymbol{\theta}$ is given by [17], [18]

$$
\mathbf{F}=\frac{2}{\sigma_{w}^{2}} \Re\left\{\frac{\partial \boldsymbol{\mu}^{H}}{\partial \boldsymbol{\theta}} \frac{\partial \boldsymbol{\mu}}{\partial \boldsymbol{\theta}^{T}}\right\}
$$

$$
\mathbf{F}=\frac{2}{\sigma_{w}^{2}}\left[\begin{array}{ccc}
\Re\left\{\boldsymbol{\Omega}_{\mathbf{f}}^{H} \boldsymbol{\Omega}_{\mathbf{f}}\right\} & -\Im\left\{\boldsymbol{\Omega}_{\mathbf{f}}^{H} \boldsymbol{\Omega}_{\mathbf{f}}\right\} & -\Im\left\{\boldsymbol{\Omega}_{\mathbf{f}}^{H} \mathbf{D}_{n} \boldsymbol{\Omega}_{\mathbf{f}} \mathbf{D}(\mathbf{h})\right\} \\
\Im\left\{\boldsymbol{\Omega}_{\mathbf{f}}^{H} \boldsymbol{\Omega}_{\mathbf{f}}\right\} & \Re\left\{\boldsymbol{\Omega}_{\mathbf{f}}^{H} \boldsymbol{\Omega}_{\mathbf{f}}\right\} & \Re\left\{\boldsymbol{\Omega}_{\mathbf{f}}^{H} \mathbf{D}_{n} \boldsymbol{\Omega}_{\mathbf{f}} \mathbf{D}(\mathbf{h})\right\} \\
-\Im\left\{\boldsymbol{\Omega}_{\mathbf{f}}^{H} \mathbf{D}_{n} \boldsymbol{\Omega}_{\mathbf{f}} \mathbf{D}(\mathbf{h})\right\}^{T} & \Re\left\{\boldsymbol{\Omega}_{\mathbf{f}}^{H} \mathbf{D}_{n} \boldsymbol{\Omega}_{\mathbf{f}} \mathbf{D}(\mathbf{h})\right\}^{T} & \Re\left\{\mathbf{D}^{H}(h) \boldsymbol{\Omega}_{\mathbf{f}}^{H} \mathbf{D}_{n}^{2} \boldsymbol{\Omega}_{\mathbf{f}} \mathbf{D}(\mathbf{h})\right\}
\end{array}\right]
$$



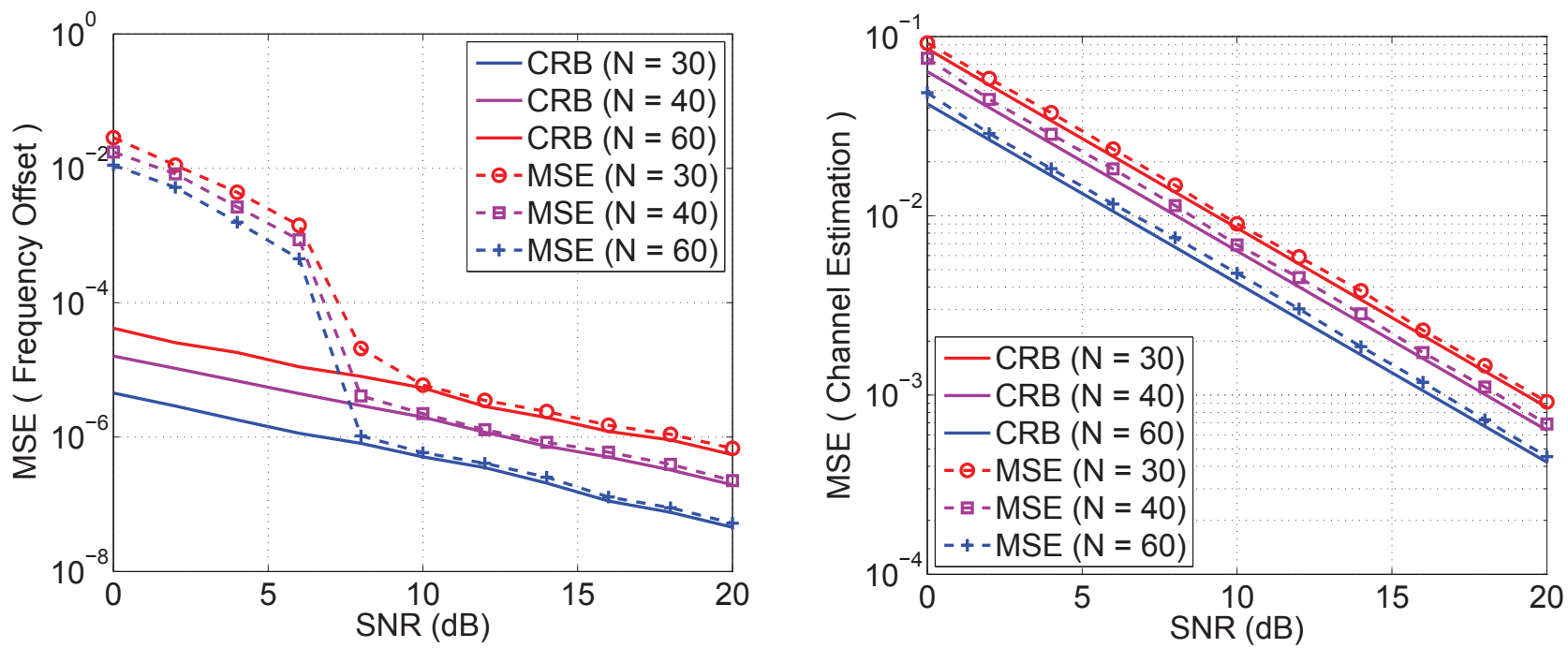

Fig. 2. MSE of frequency offset estimation as a function of SNR (dB), Fig. 3. MSE of channel estimation as a function of SNR (dB), with training with training sequence length $N=30,40,60$ respectively. CRB in (19) is sequence length $N=30,40,60$ respectively. CRB in (18) is plotted as a plotted as a reference.

where $\Re\{\cdot\}$ denotes the real part.

The FIM in (16) for joint channel and frequency offsets estimate can be viewed as a special case of the one derived for Multiple Input Multiple Output (MIMO) systems in [8]. Keeping in mind the single output node and multiple single antenna input nodes, FIM can be written as (17), where $\mathbf{D}_{n}=$ $2 \pi \times \operatorname{diag}(1,2, \ldots, N)$ and $\mathbf{D}(\mathbf{h})=\operatorname{diag}(\mathbf{h})$.

Using the definition of $\operatorname{CRB}(\boldsymbol{\theta})=\mathbf{F}^{-1}$, the CRB for the estimation of $\mathbf{h}$ is given by [8]

$$
\mathrm{CRB}(\mathbf{h})=\frac{\sigma_{w}^{2}}{2}\left\{2 \mathbf{U}^{-1}+\mathbf{U}^{-1} \mathbf{T}[\Re(\mathbf{W})]^{-1} \mathbf{T}^{H} \mathbf{U}^{-1}\right\}
$$

and $\mathrm{CRB}$ for the estimation of $\mathbf{f}$ is given by

$$
\operatorname{CRB}(\mathbf{f})=\frac{\sigma_{w}^{2}}{2}\left\{\Re\left(\mathbf{V}-\mathbf{T}^{H} \mathbf{U}^{-1} \mathbf{T}\right)\right\}
$$

where $\mathbf{U} \triangleq \mathbf{\Omega}^{H} \boldsymbol{\Omega}, \mathbf{T} \triangleq \boldsymbol{\Omega}^{H} \mathbf{D}_{n} \boldsymbol{\Omega} \mathbf{D}(\mathbf{h}), \quad \mathbf{V} \triangleq$ $\mathbf{D}^{H}(\mathbf{h}) \boldsymbol{\Omega}^{H} \mathbf{D}_{n}^{2} \boldsymbol{\Omega} \mathbf{D}(\mathbf{h})$ and $\mathbf{W} \triangleq \mathbf{V}-\mathbf{T}^{H} \mathbf{U}^{-1} \mathbf{T}$.

(18) and (19) show that the CRB for the estimation of $\mathbf{h}$ depends on $f$ and the CRB for the estimation of $f$ depends on $\mathbf{h}$ through the matrix $\mathbf{D}(\mathbf{h})$ respectively. Thus, there is no decoupling between the estimation errors corresponding to the channel and frequency parameters and joint CRB formulation is necessary to study the lower bound on the estimation of multiple frequency offsets. Note that since the CRB for the estimation of $\mathbf{h}$ depends on the matrix $\mathbf{D}(\mathbf{h})$, we average (18) and (19) over 2000 realizations of Rayleigh fading channel.

\section{Vi. Simulation Results}

In this section, we present simulation results to verify the performance of our proposed equalizer. We assume both relays employ Quadrature Phase Shift Keying (QPSK) modulation with the full rate $2 \times 1$ Alamouti STBC to cooperatively transmit the data. The propagation channels are i.i.d. block Rayleigh flat-fading channels. The frequency offsets are assumed to be uniformly distributed in the range $(-0.5,0.5)$, i.e. reference.

the full acquisition range. We generate the training sequence as $\left[\exp \left(-j \phi_{1}\right), \ldots, \exp \left(-j \phi_{N}\right)\right]$, where $\phi_{i}$ is uniformly distributed between $[-\pi, \pi]^{2}$. We analyze the system performance for different lengths of training sequence, i.e., $N=30,40$ and 60. We use the Mean Square Error (MSE) of channel and frequency offsets and mean Bit Error Rate (BER) as figures of merit. All the simulation results are averaged over $L=2500$ Monte Carlo runs.

\section{A. Mean Square Error of Frequency Offsets Estimation}

Fig. 2 shows the Mean Square Error (MSE) of frequency offsets estimation for different values of signal-to-noise ratio (SNR). The MSE of the multiple frequency offsets is calculated as, MSE $=\frac{1}{2 L} \sum_{k=1}^{2} \sum_{\ell=1}^{L}\left(f_{k}(\ell)-\hat{f}_{k}(\ell)\right)^{2}$, where $L$ is the number of Monte Carlo simulation runs for each value of SNR, $f_{k}(\ell)$ is the introduced random frequency offset between Relay $R_{k}$ and destination for $\ell^{\text {th }}$ simulation and $\hat{f}_{k}(\ell)$ is the estimate of that frequency offset for $\ell^{\text {th }}$ simulation. The CRB for frequency offset estimation in (19) is plotted as reference. We can see in Fig. 2 that the MSE decreases by increasing SNR and increasing the number of symbols in the training sequence. The MSE achieves the lower bound after approximately $\mathrm{SNR}=7 \mathrm{~dB}$, which validates maximum likelihood estimator with reduced complexity in (9).

\section{B. Mean Square Error of Channel Estimation}

Fig. 3 shows the Mean Square Error (MSE) of channel estimation for different values of SNR. The MSE of multiple channels between the 2 relays and the destination is calculated as, MSE $=\frac{1}{2 L} \sum_{k=1}^{2} \sum_{\ell=1}^{L}\left(h_{k}(\ell)-\hat{h}_{k}(\ell)\right)^{2}$, where $L$ is the number of Monte Carlo simulation runs for each value of SNR, $h_{k}(\ell)$ is a random realization of Rayleigh fading channel between Relay $R_{k}$ and destination for $\ell^{\text {th }}$ simulation and $\hat{h}_{k}(\ell)$

\footnotetext{
${ }^{2}$ Note that this sequence may not be optimal, but the problem of optimal training design is beyond the scope of this paper, see [17].
} 


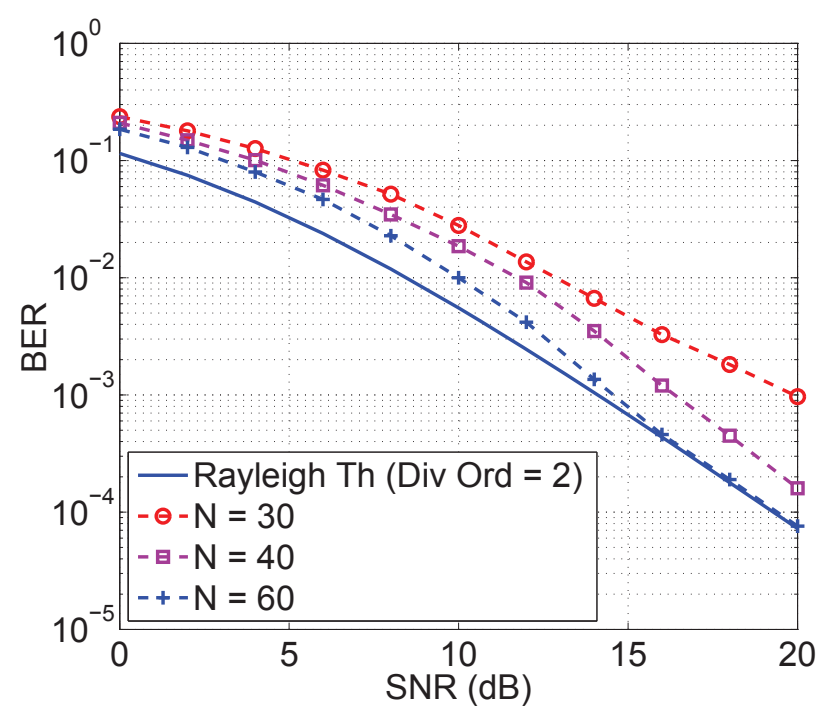

Fig. 4. BER of the proposed receiver as a function of SNR (dB), with training sequence length $N=30,40,60$ respectively.

is the estimate of that channel for $\ell^{\text {th }}$ simulation. The CRB for channel estimation in (18) is plotted as reference. We can see in Fig. 3 that the MSE decreases by increasing SNR and increasing the number of symbols in the training sequence. The MSE achieves the lower bound for the whole range of considered SNR, which validates maximum likelihood estimator with reduced complexity in (7).

\section{Bit Error Rate Performance and Comparison with Existing Results}

Fig. 4 shows the BER of the cooperative communication system as a function of SNR. The theoretical curve for Rayleigh fading channel with diversity order $=2$ is plotted as a reference. It can be seen that at low SNR values (approximately less then $7 \mathrm{~dB}$ ), the simulation results are further away from the theoretical result. This is due to the larger values of MSE of frequency offset estimation as shown in Fig. 2. The slope of the BER results achieves the theoretical diversity order of 2 for $N=40$ and 60 , which confirms the correct working of the proposed equalizer in (14). In addition, for training sequence length $N=60$ the simulated BER achieves the theoretical BER value after approximately $\mathrm{SNR}=16 \mathrm{~dB}$.

The MSE of MCFO estimation algorithm in [11], which is shown to outperform existing algorithms, achieves or approaches the CRB approximately above $10 \mathrm{~dB}$ SNR and the BER matches the perfectly synchronized case at $15 \mathrm{~dB}$ SNR, which is similar to our case. Note that [11] adopts the equalizer design in [3] for MCFO compensation. The BER performance of existing equalizer designs in [3]-[7] is almost similar to that of our proposed equalizer but they assume a small range of normalized frequency offset differences among relay nodes (typically less than \pm 0.1 ) and perfect MCFO and channel estimation. Our design has the advantage that it can work for the full acquisition range of the multiple carrier frequency offsets and we use the maximum likelihood estimates of these parameters in the equalizer design.

\section{CONCLUSIONS}

By exploiting maximum likelihood decoding with alternating projection, we have proposed an efficient estimator for multiple carrier frequency offsets and Rayleigh fading channels in cooperative communication systems. Based on these estimates, our proposed equalizer uses matrix inverse technique to estimate the symbols. We have formulated the Cramér Rao Bound (CRB) and showed that the MSE of MCFO and achieves the CRB above $7 \mathrm{~dB}$ SNR while the MSE of channel estimation achieves the CRB for all SNR values. In addition the Bit Error Rate (BER) performance of our equalizer fully achieves the spatial diversity gain of space-time block codes. The proposed equalizer design can be applied in decode and forward cooperative communication systems even if the frequency offsets are large.

\section{REFERENCES}

[1] A. Sendonaris, E. Erkip, and B. Aazhang, "User cooperative diversity - part I: system description; part II: implementation and performance analysis," IEEE Trans. Commun., vol. 51, pp. 1927-1948, Nov. 2003.

[2] X. Li, Y. C. Wu, and E. Serpedin, "Timing synchronization in decodeand-forward cooperative communication systems." IEEE Trans. Signal Process., vol. 57, no. 4, pp. 1444-1455, Apr. 2009.

[3] H. Wang, X.-G. Xia, and Q. Yin, "Computationally efficient equalization for asynchronous cooperative communications with multiple frequency offsets," IEEE Trans. Wireless Commun., vol. 8, pp. 648-655, 2009.

[4] Z. Li, D. Qu, and G. Zhu, "An equalization technique for distributed STBC-OFDM system with multiple carrier frequency offsets," in Proc. IEEE WCNC, 2006.

[5] F. Tian, X.-G. Xia, and P. C. Ching, "Signal detection in a cooperative communication system with multiple CFOs by exploiting the properties of space-frequency codes," in Proc. IEEE ICC, 2008.

[6] D. Veronesi and D. L. Goeckel, "Multiple frequency offset compensation in cooperative wireless systems," in Proc. IEEE GLOBECOM, 2006.

[7] Y. Zhang and J. Zhang, "Multiple CFOs compensation and BER analysis for cooperative communication systems," in Proc. IEEE WCNC, 2009.

[8] O. Besson and P. Stoica, "On parameter estimation of MIMO flat-fading channels with frequency offsets," IEEE Trans. Signal Process., vol. 51, no. 3, pp. 602-613, Mar. 2003.

[9] Y. Yao and T. Ng, "Correlation based frequency offset estimation in MIMO system," in Proc. IEEE VTC, 2003.

[10] T. Pham, A. Nallanathan, and Y. Liang, "Joint channel and frequency offset estimation in distributed MIMO flat-fading channels," IEEE Trans. Wireless Commun., vol. 7, no. 2, pp. 648-656, Feb. 2008.

[11] H. Mehrpouyan and S. D. Blostein, "Bounds and algorithms for multiple frequency offset estimation in cooperative networks," IEEE Trans. Wireless Commun., vol. 10, no. 4, pp. 1300-1311, Apr. 2011.

[12] Y. Yu, A. P. Petropulu, H. V. Poor, and V. Koivunen, "Blind estimation of multiple carrier frequency offsets," in Proc. IEEE PIMRC, 2007.

[13] X. Liu, J. Kountouriotis, A. Petropulu, and K. R. Dandekar, “ALOHA with collision resolution (ALOHA-CR): Theory and software defined radio implementation," IEEE Trans. Signal Process., vol. 58, no. 8, pp. 4396-4410, Aug. 2010.

[14] M.-O. Pun, M. Morell, and C.-C. J. Kuo, "Maximum likelihood synchronization and channel estimation for OFDMA uplink transmissions," IEEE Trans. Commun., vol. 54, no. 4, pp. 726-736, Apr. 2006.

[15] H. Meyr, M. Moeneclaey, and S. Fechtel, Digital Communication Receivers, Synchronization, Channel Estimation, and Signal Processing, J. Proakis, Ed. Wiley Series in Telecom. and Signal Processing, 1998.

[16] S. M. Alamouti, "A simple transmit diversity technique for wireless communications," IEEE J. Sel. Areas Commun., vol. 16, no. 8, pp. 14511458, Oct. 1998.

[17] P. Stoica and O. Besson, "Training sequence design for frequency offset and frequency-selective channel estimation," IEEE Trans. Commun., vol. 51, no. 11, pp. 1910-1917, Nov. 2003.

[18] H. L. V. Trees and K. L. Bell, Bayesian Bounds for Parameter Estimation and Nonlinear Filtering / Tracking, M. E. El-Hawary, Ed. Wiley interscience, 2007. 